\section{Energy Focus \\ Lithium-ion intercalation visualized in situ with new atomic-scale imaging technique}

Tntercalation in rechargeable lithium-ion batteries - the process where lithium ions pack themselves in between adjacent two-dimensional layers of an electrode material - is integral to the operation of the batteries. The complete dynamics of this process have not yet been visualized. M. Azhagurajan from Tohoku University, Y.-G. Kim from the California Institute of Technology, and their colleagues have now succeeded in this goal, as reported in their article published recently in the Journal of the American Chemical Society (doi:10.1021/jacs.5b11849).

The major technological advance achieved by the team was the development of a combined laser confocal microscopydifferential interference microscopy (LCMDIM) imaging technique, which enabled single atomic step heights down to tenths of nanometers to be resolved. Using this technique, the researchers examined the intercalation and deintercalation of lithium within $\mathrm{MoS}_{2}$ layers, recording and analyzing contrasts in the images. They found that intercalation occurs layer by layer in a stepwise manner; beginning at the layer's edge, a lithium ion enters between $\mathrm{MoS}_{2}$ layers. The ion then accommodates a second ion by shifting one unit cell toward the interior of the layer. These two intercalated ions then shift one unit cell toward the interior to accommodate a third ion. This process continues until a layer of $\mathrm{MoS}_{2}$ is almost completely intercalated with $\mathrm{Li}$ ions before intercalation in the subsequent layer begins. Deintercalation proceeds in a similar way.

The layer-by-layer process of intercalation and deintercalation gives rise to a distinct phase separation between

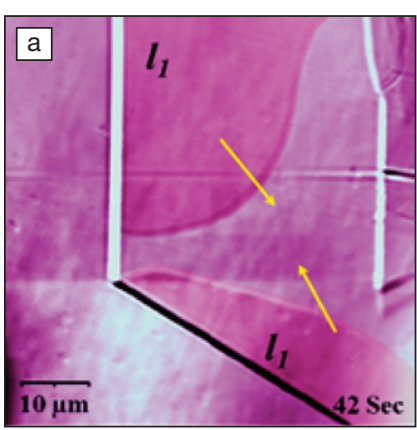

(a) Laser confocal microscope combined with a differential interference contrast microscope image of $\mathrm{Li}$-ion intercalation of the first intercalated layer of $\mathrm{MoS}_{2}$ where the time marked in the image shows the start of the appearance of the new domain. The image was obtained at $1.25 \mathrm{~V}$ versus $\mathrm{Li}$, and taken in an area of $70 \times 70 \mu \mathrm{m}^{2}$. (b,c) Schematic of $\mathrm{Li}$-ion intercalation within $\mathrm{MoS}_{2}$ layers along the $b$-axis. The first layer underneath the selvedge is intercalated first to (near) completion prior to the intercalation of the second layer. A solvated $\mathrm{Li}$ ion is initially intercalated at the steps. (d) A row of four intercalated $\mathrm{Li}$ ions takes in an incoming $\mathrm{Li}$ ion, making the row with five ions. Credit: Journal of the American Chemical Society.
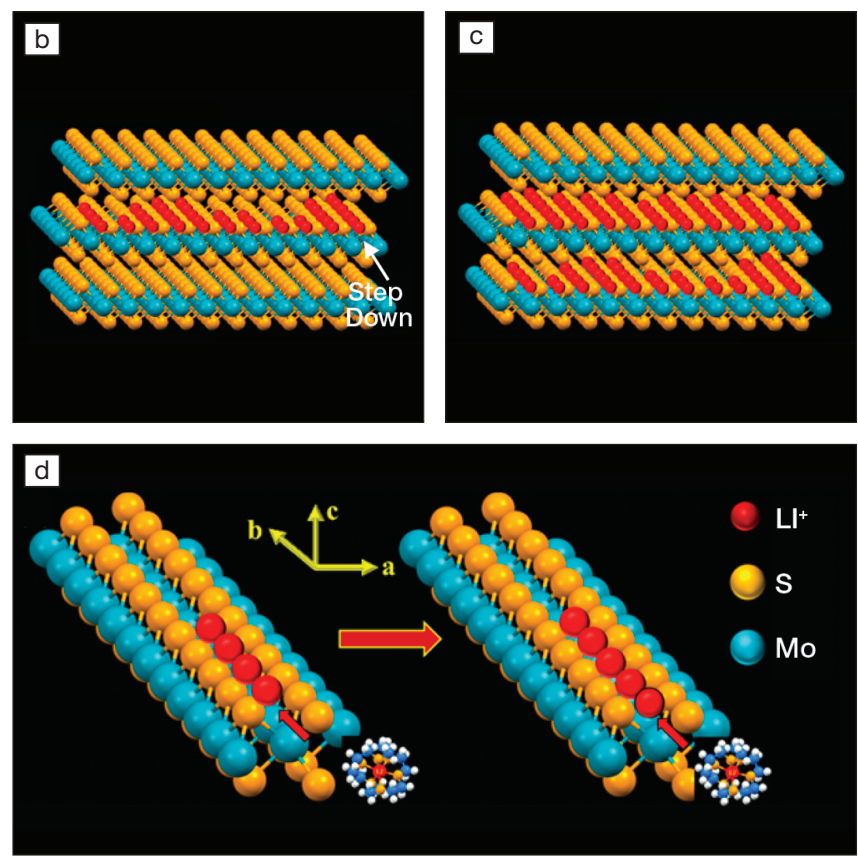

the intercalated and non-intercalated regions. Using the LCM-DIM in situ dynamic visualization technique, the team was able to see the intercalated region expand as a front moving through the material, much like a layer of water expands to cover a smooth surface. With application of greater current, net-like disruptions occurred in the layer surface.
These reticular regions later developed into cracks in the $\mathrm{MoS}_{2}$ surface.

The LCM-DIM opens the door to detailed analysis of atomic-scale surface phenomena that are accompanied by changes in optical properties, potentially ushering in novel discoveries and advances in battery technology.

Antonio Cruz
Glassy poly(lactide) toughened with diblock copolymer micelles

rowing environmental concerns Jare calling for nondegradable petroleum-derived plastics to be replaced with biodegradable, more sustainable options. Poly(L-lactide) (PLLA), a product of polymerization of L,L-lactide and a different form of poly(lactide) (PLA) by virtue of its chirality, is one of the many viable alternatives with high tensile strength and high elastic modulus, but its intrinsically brittle characteristic must be overcome for applications requiring toughness, impact resistance, and optical clarity. A research group with the Center for Sustainable Polymers at the University of Minnesota have now successfully toughened PLLA by 